\title{
A mixed-methods systematic review protocol to examine the use of physical restraint with critically ill adults and strategies for minimizing their use
}

Louise Rose $^{1,2,3,4^{*}}$, Craig Dale ${ }^{2}$, Orla M. Smith ${ }^{5}$, Lisa Burry ${ }^{6,7}$, Glenn Enright ${ }^{2}$, Dean Fergusson ${ }^{8,9}$, Samir Sinha ${ }^{7,10,11,12}$, Lesley Wiesenfeld ${ }^{7}$, Tasnim Sinuff ${ }^{1,10}$ and Sangeeta Mehta ${ }^{7,10}$

\begin{abstract}
Background: Critically ill patients frequently experience severe agitation placing them at risk of harm. Physical restraint is common in intensive care units (ICUs) for clinician concerns about safety. However, physical restraint may not prevent medical device removal and has been associated with negative physical and psychological consequences. While professional society guidelines, legislation, and accreditation standards recommend physical restraint minimization, guidelines for critically ill patients are over a decade old, with recommendations that are non-specific. Our systematic review will synthesize evidence on physical restraint in critically ill adults with the primary objective of identifying effective minimization strategies.

Methods: Two authors will independently search from inception to July 2016 the following: Ovid MEDLINE, CINAHL, Embase, Web of Science, Cochrane Library, PROSPERO, Joanna Briggs Institute, grey literature, professional society websites, and the International Clinical Trials Registry Platform. We will include quantitative and qualitative study designs, clinical practice guidelines, policy documents, and professional society recommendations relevant to physical restraint of critically ill adults. Authors will independently perform data extraction in duplicate and complete risk of bias and quality assessment using recommended tools. We will assess evidence quality for quantitative studies using the Grading of Recommendations Assessment, Development and Evaluation (GRADE) approach and for qualitative studies using the Confidence in the Evidence from Reviews of Qualitative Research (CERQual) guidelines. Outcomes of interest include (1) efficacy/effectiveness of physical restraint minimization strategies; (2) adverse events (unintentional device removal, psychological impact, physical injury) and associated benefits including harm prevention; (3) ICU outcomes (ventilation duration, length of stay, and mortality); (4) prevalence, incidence, patterns of use including patient and treatment characteristics and chemical restraint; (5) barriers and facilitators to minimization; (6) patient, family, and healthcare professional perspectives; (7) professional society-endorsed recommendations; and (8) evidence gaps and research priorities.
\end{abstract}

(Continued on next page)

\footnotetext{
* Correspondence: louise.rose@utoronto.ca

${ }^{1}$ Department of Critical Care Medicine, Sunnybrook Health Sciences Centre,

2075 Bayview Ave, Toronto M4N 3M5, Canada

${ }^{2}$ Lawrence S. Bloomberg Faculty of Nursing, University of Toronto, 155

College St., Toronto M5T 1P8, Canada

Full list of author information is available at the end of the article
} 
(Continued from previous page)

Discussion: We will use our systematic review findings to produce updated guidelines on physical restraint use for critically ill adults and to develop a professional society-endorsed position statement. This will foster patient and clinician safety by providing clinicians, administrators, and policy makers with a tool to promote minimal and safe use of physical restraint for critically ill adults.

Systematic review registration: PROSPERO CRD42015027860

Keywords: Physical restraint, Patient safety, Intensive care, Mechanical ventilation, Systematic review

\section{Background}

Physical restraint is highly prevalent in the critically ill, with up to $75 \%$ of mechanically ventilated adults having physical restraints applied at least once during their intensive care unit (ICU) admission [1]. Annually, an estimated 13 million people are admitted to ICUs worldwide [2] with approximately $30 \%$ requiring mechanical ventilation $[3,4]$. While acknowledging variation in international physical restraint practice [5], some reports indicate the prevalence of physical restraint is greater than $70 \%$ of mechanically ventilated critically ill patients [6] who are in turn at risk of associated adverse physical and psychological consequences annually. While government legislation, hospital accreditation standards, and professional society guidelines recommend physical restraint minimization [7], available guidelines on alternative strategies to physical restraint for critically ill adults [8] are outdated and are not specific regarding recommendations to support the minimization of physical restraint use.

In the ICU, physical restraint is frequently used for clinician concerns for patient and/or clinician safety including prevention of accidental removal of medical devices (e.g. endotracheal tubes, central venous catheters) [5]. However, evidence does not uniformly or persuasively indicate that physical restraint effectively reduces the risk of accidental device removal [9-11]. For example, a multi-centre study conducted in 39 hospitals in the USA found that $44 \%$ of hospitalized patients were restrained at the time of device removal [12] and multiple studies of critically ill patients indicate they were restrained at the time of self-extubation or other device removal [10-15]. A recent case-control study with matching by mechanical ventilation duration further reported that physically restrained patients were in fact five times more likely to experience unplanned extubation [16].

Despite the intention to promote patient and clinician safety, physical restraint can adversely impact patient safety resulting in detrimental physical and psychological consequences [17-22]. Negative physical consequences include tissue injury [23], immobility, pressure ulcers, and nosocomial infection [24]. Negative psychological consequences include agitation, disorientation, psychological trauma, and delirium, a serious and potentially fatal complication of critical illness [18-20]. Physical restraint without sedation has been associated with delusional memories and posttraumatic stress disorder in ICU survivors [21, 22]. Finally, physical restraint is often not removed expeditiously when agitation has resolved, thereby negatively impacting patient safety.

Given the recognized adverse physical and psychological consequences of physical restraint and the lack of efficacy for preventing device removal, government legislation, hospital accreditation standards, and professional society guidelines all advocate for minimization of physical restraint across all healthcare settings [8, 25, 26]. However, these recommendations lack specificity on how to do this, resulting in institutional and healthcare team practice variation with respect to triggers for physical restraint utilization and removal, use of alternatives, documentation standards, and patient monitoring during physical restraint.

The identification of effective physical restraint minimization strategies is an immediate clinical and research imperative and has implications in terms of patient- and family-reported experience and outcome measures. To address this need, we will conduct a systematic review with the overall objective of producing an updated practice guideline on physical restraint for critically ill adults and developing a professional societyendorsed position statement. Our objectives include synthesizing quantitative studies of the (1) efficacy and effectiveness of physical restraint minimization strategies; (2) adverse events (unintentional device removal, psychological impact, physical injury) and associated benefits including harm prevention; (3) ICU outcomes (ventilation duration, length of stay, and mortality); and (4) prevalence, incidence, and patterns of use including patient and treatment characteristics and chemical restraint. Additionally, we will synthesize quantitative and qualitative evidence on barriers and facilitators to minimization and patient, family member, and ICU healthcare professional perspectives and identify professional society-endorsed recommendations relevant to ICU patients and evidence gaps and research priorities.

\section{Methods}

This review protocol was prepared using the Preferred Reporting Items for Systematic Reviews and Meta-Analyses 
Protocol (PRISMA-P) guidelines [27]. We completed the PRISMA-P checklist (Additional file 1). We registered the protocol on International Prospective Register of Systematic Reviews (PROSPERO) (CRD42015027860).

\section{Data sources and search strategy}

We created a preliminary search strategy (Additional file 2) as an iterative process under the guidance of an experienced information specialist. For the purposes of this systematic review and guideline update, we have defined physical restraint as mechanical devices that restrict patients' movements [8]. Prior to execution of the search, a second information specialist used the Peer Review for Electronic Search Strategies (PRESS) template $[28,29]$ to review the search strategy. We will search the following electronic databases from inception to July 2016: Ovid MEDLINE $^{\oplus}$ (OvidSP), Ovid MEDLINE ${ }^{\bullet}$ In-Process \& Other Non-Indexed Citations, CINAHL (EBSCOhost), Embase (OvidSP), and ISI Web of Science and Conference Proceedings. We will search for systematic reviews within the Cochrane Library, PROSPERO, and the Joanna Briggs Institute and will search for unpublished studies and ongoing trials on the http://apps.who.int/trialsearch website. We will search major guideline sites (e.g. CMA Infobase, National Guideline Clearinghouse) and websites of relevant professional societies and conduct general searches using tools such as Google Scholar, ScienceWatch, and MSN. We will examine reference lists of relevant studies and will contact corresponding authors for additional published or unpublished work.

Search strategies will utilize a combination of controlled vocabulary (e.g. 'Intensive Care Units', 'Critical Care,' 'Restraint, Physical') and keywords (e.g. ICU, highdependency unit, restraint). Vocabulary and syntax will be adjusted across databases. We will remove animalonly studies and opinion pieces (e.g. editorials, letters). We will not impose language restrictions in our database searches but will only seek to obtain policy documents written in English. As we are including a range of study designs, we will not apply study-specific filters.

\section{Study eligibility criteria}

We will include a range of study designs, including qualitative studies describing perspectives/restraint experience as well as clinical practice guidelines, policy documents, and professional society recommendations relevant to critically ill adults. To determine the efficacy/effectiveness and safety of physical restraint minimization strategies, we will include parallel group randomized controlled trials including crossover trials, quasi-randomized trials (such as alternate allocation based on day of the week), and non-randomized studies including non-randomized controlled trials; controlled before and after studies; historically controlled studies; and retrospective or prospective cohort studies that include a control group. Inclusion of evidence from qualitative studies that explore the experience of patients and family members receiving interventions and healthcare providers delivering the intervention, as well as studies that evaluate barriers and facilitators to implementation of interventions, have an important role in maximizing the value of a systematic review to inform clinical practice, policy, and decision-making [30-32].

\section{Population}

Our population of interest is adults admitted to a high intensity care setting including ICUs, specialized weaning centres, and high-dependency units. We will include all high intensity care setting patient populations including those admitted for medical, surgical, neurological, cardiac, and trauma diagnoses. We will exclude studies of physical restraint use in patients admitted to other acute care settings such as the emergency department, postoperative recovery units, hospital floors/wards, psychiatry, and in long-term care settings.

\section{Intervention}

For studies examining the efficacy and safety of physical restraint minimization, interventions will include any strategy used for the purpose of decreasing the frequency and duration of physical restraint. Such strategies may comprise pharmacological and non-pharmacological interventions used to alleviate anxiety and optimize analgesia and alternatives to ensure patient safety such as sitters or observers. Patients may receive chemical restraint in the form of sedatives and neuromuscular blockers. For noninterventional and qualitative studies, we will include data from patients that are not physically restrained during admission to a high care intensity setting.

\section{Comparators}

For studies examining the efficacy and safety of physical restraint minimization and non-interventional and qualitative studies, comparator groups will receive usual care that comprises physical restraint methods such as wrist restraints, ankle restraints, chest belt, bed rails, vest restraints, mitts or mittens, four-point restraints, and five-point restraints.

\section{Outcomes}

Our primary outcome of interest is the efficacy/effectiveness of physical restraint minimization strategies defined as the proportion of patients physically restrained. Secondary outcomes include (1) adverse events including unintentional device removal (endotracheal and tracheostomy tubes, catheters, intravenous lines, feeding tubes, and wound drains); delirium; severe agitation; psychological impact including anxiety, depression, and posttraumatic stress disorder (quantitative measures or 
qualitative description); physical injury resulting from restraint; and associated benefits including harm prevention; (2) ICU outcomes including duration of mechanical ventilation, ICU length of stay, and mortality; (3) prevalence, incidence, and patterns of physical restraint use including patient and treatment characteristics and chemical restraint; (4) barriers and facilitators to minimization; and (5) patient, family member, and healthcare professional perspectives on physical restraint. Additionally, we will identify recommendations for restraint minimization endorsed by professional societies and accreditation and regulatory agencies and evidence gaps and priorities for research.

\section{Screening and data extraction}

Two authors (LR, GE) will independently screen search results against eligibility criteria. Full-text publications of all potentially relevant articles, selected by either author, will be retrieved and examined for eligibility. We will resolve any disagreements though discussion and refer to an independent arbiter (CD) if required. We will use the reference management software EndNote X7 [33] to remove duplicates and sort exclusions and inclusions using the create group function. We will document the search strategy and study selection process using a PRISMA flow diagram [34].

Teams of two authors will independently extract data from selected studies on key components addressing our research questions including features of study design, patient characteristics, prevalence and incidence, study outcomes, benefits and adverse events, perceptions, and recommendations using designed data extraction forms, iteratively refined based on pilot data extraction. We have developed three data extraction forms for (1) studies with a control group, (2) studies without a control group, and (3) studies with a qualitative design. Due to inherent difficulties with determining what constitute findings in a qualitative research [35], we will extract category- or theme-level evidence from the findings or results section of the included papers only. Extraction will be checked and any disagreement resolved by a third author. We will confirm all data extraction, and where necessary, discrepancies will be resolved by an independent arbiter (LR).

\section{Risk of bias and quality assessment}

We will critically appraise included papers in duplicate and independently for risk of bias. For randomized and quasi-randomized trials, we will use the domain-based evaluation recommended by The Cochrane Handbook. [36] These domains include the following: random sequence generation, allocation concealment, blinding, incomplete outcome data, selective reporting, and other biases. For each domain, we will assign a judgement regarding the risk of bias as 'high risk of bias', 'low risk of bias', or 'unclear risk of bias' [36]. Once we achieve consensus on the quality assessment of the six domains for eligible studies, we will assign them to the following categories: low risk of bias: low risk scored in all domains; high risk of bias: high risk scored in one or more domains; and unclear risk of bias: unclear risk scored in one or more domains (and no domain at the high risk of bias). For quality assessment of observational studies, we will use the SIGN checklists for cohort and case-control studies as recommended by the Quality Assessment Tools Project Report [37]. We will construct a 'risk of bias' table to present the results.

For qualitative studies, we will assess study quality using the multi-dimensional concept of quality recommended by the Cochrane Quality and Intervention Methods Group [38] that includes (1) quality of reporting, e.g. explicitness in reporting all study aspects; (2) methodological rigour, e.g. validity and reliability of study design and process; and (3) overall conceptual depth and breadth, e.g. if stated study aims, rationale, or theory (if the study is explicitly theoretically informed) are reflected in the study design, process, and findings. Two authors will independently appraise study quality using the 10 questions of the 2014 Critical Appraisal Skills Programme (CASP) quality assessment tool for qualitative studies [39]. Each of these 10 questions will be answered as 'yes', 'no', or 'unclear'. The CASP tool does not consider the more conceptual or theoretical aspects of qualitative studies; the same two authors will independently appraise study quality using the seven criteria outlined by Popay et al. [40]. These seven criteria include (1) illumination of subjective meaning, (2) adaptation and responsiveness of research design, (3) sample provides appropriate knowledge, (4) description sufficiently detailed, (5) sources of knowledge compared and contrasted, (6) shift from description to analysis and interpretation, and (7) claims for generalizability. We will present quality assessments in tabular format.

\section{Approach to evidence synthesis}

Search results will be summarized in a Preferred Reporting Items for Systematic Reviews and MetaAnalyses (PRISMA) study flow diagram [41]. We will categorize studies according to study design and then summarize study characteristics within these categories using frequencies and percentages for categorical variables and means and standard deviations or median and interquartile ranges for continuous variables depending on data distribution. We will generate tables reporting types and frequency of adverse outcomes associated with physical restraint, benefits associated with physical restraint, prevalence and incidence overall and according to country, characteristics of physically restrained patients, and strategies 
used to minimize physical restraint. We anticipate qualitative data will describe patient, family, and healthcare provider perspectives on physical restraint use as well as barriers and facilitators to physical restraint minimization. We will generate tables of author-reported categories, themes, and subthemes as well as existing professional society recommendations related to physical restraint use. We will undertake thematic content analysis [42] to identify common categories and themes within these categories. We will similarly examine professional society guidelines and recommendations to identify commonalities across guidelines.

\section{Measurement of treatment effect}

If we identify sufficient studies evaluating interventions to minimize physical restraint, we will perform metaanalyses for the following outcomes: proportion of patients physically restrained, duration of mechanical ventilation, ICU length of stay, mortality, and adverse events. If we identify fewer than three randomized or quasi-randomized trials, we will include non-randomized studies in this meta-analysis.

For binary outcomes, we will calculate risk ratios (RRs) with 95\% confidence intervals (CIs) using a random effects model. For continuous variables, we will calculate a pooled difference of means with $95 \%$ confidence intervals using a DerSimonian Laird random effects model. If the mean (standard deviation, SD) is not reported or unavailable from the study authors, we will use the median (IQR) to approximate the mean using the method described by Hozo and colleagues [43] and will calculate an approximate SD from the IQR [36]. If required, we will $\log$ transform skewed data. We will consider a two-sided $P$ value $<0.05$ to be significant. We will use RevMan 5.3 software to conduct meta-analyses. If we identify insufficient studies for meta-analyses, we will provide a descriptive qualitative synthesis.

\section{Subgroup and sensitivity analysis}

If we identify sufficient studies of interventions to minimize physical restraint, we will perform subgroup analyses considering intervention type and patient characteristics such as age and admission category (medical, surgical, cardiac, neurological, trauma). We will conduct two sensitivity analyses, one excluding non-randomized studies and the second excluding studies rated as high risk of bias.

\section{Unit of analysis issues}

We will use individual study participants as the unit of analysis. If we identify multi-arm studies, we will combine groups to create a single pairwise comparison as recommended by the Cochrane Handbook for Systematic Reviews of Interventions [36]. If combining groups is not possible or feasible, we will select only one treatment group receiving the most intense level of intervention to minimize physical restraint and a control group receiving the least intervention from each study.

\section{Dealing with missing data}

We (LR) will attempt to contact study authors for unreported data or clarification of study methods using a maximum of three e-mails. If data remains unavailable, we will analyse the available data and report the potential impact of missing data in the discussion section.

\section{Assessment of heterogeneity}

If we identify sufficient studies evaluating interventions to minimize physical restraint, we will assess clinical and methodological heterogeneity with forest plots and chisquare tests $(P<0.05$ represents significant heterogeneity) and using the $I^{2}$ statistic, which represents the percentage of variability across studies attributable to heterogeneity rather than chance [36]. If the $I^{2}$ statistic is $>50 \%$ statistic, indicating moderate to substantial heterogeneity, we will assess the type and sources of heterogeneity (clinical and methodological) such as ICU patient type and physical restraint minimization intervention used.

\section{Publication bias}

If sufficient studies are identified, we will assess for publication bias by constructing a funnel plot of all studies included in any analysis [44] as well as using Egger's regression test [45] and the Macaskill test [46, 47].

\section{Assessing confidence in evidence}

We will use the Grading of Recommendations Assessment, Development and Evaluation (GRADE) approach [48] to assess the confidence in the evidence of effectiveness arising from studies evaluating interventions to minimize physical restraint for the following outcomes: proportion of patients physically restrained, duration of mechanical ventilation, ICU length of stay, mortality, and adverse events. We will present our GRADE assessments in a summary of findings table.

We will use the Confidence in the Evidence from Reviews of Qualitative Research (CERQual) approach to assess confidence in qualitative evidence synthesis [49]. This relatively new approach makes judgements on four components: (1) methodological limitations of included studies, (2) relevance of contributing studies to the research question, (3) coherence of study findings, and (4) adequacy of the data supporting the study findings. Judgements related to the four CERQual components will be summarized in a CERQual Qualitative Evidence Profile (Additional file 3) [49]. Two authors will independently assess each CERQual component individually 
and across the four components to make a final assessment. We will rate overall assessment of confidence as high, moderate, low, or very low and provide a reason for this judgement. We will assign high confidence if it is highly likely, moderate confidence if likely, low confidence if it is possible, and very low confidence if it is not clear that the review finding is a reasonable representation of the phenomenon of interest [49]. We will present our CERQual findings in a summary of qualitative findings table.

Tabulated data, quality of evidence, and confidence in evidence assessment findings will be provided to an interprofessional and international guideline development group to develop recommendations for inclusion in an updated practice guideline on physical restraint for critically ill adults.

\section{Discussion}

While nursing homes and psychiatric units have seen a significant reduction in physical restraint use over the last two decades, use amongst the critically ill remains extremely common and may have detrimental physical and psychological consequences [12, 17-22]. Data from a cohort of hospitals in the USA indicate that over 2 years, approximately 27,000 patients were physically restrained daily, with most physical restraint use (56\%) occurring in ICUs, despite this care location accounting for only $16 \%$ of overall hospital days [50]. In a 2013 survey of 121 French ICUs, physical restraints were used at least once in more than $50 \%$ of mechanically ventilated patients. Moreover, ventilated patients in most ICUs (65\%) were restrained for greater than half the ventilation duration [51]. Interestingly, practice variation exists with some countries declaring minimal to no physical restraint use in the ICU [5]. In a binational comparison study, physical restraint prevalence in a sample of three US ICUs was $39 \%$, compared with $0 \%$ in two Norwegian ICUs, though Norwegian patients were more sedated [52]. Therefore, physical restraint is highly prevalent in the critically ill patient population with some evidence of adverse consequences for patients. As such, identification of effective physical restraint minimization strategies is an immediate clinical and research imperative with the potential to influence patient- and family-reported experience and outcomes.

We will use our mixed-methods systematic review findings to develop a professional society-endorsed position statement and to update the 2003 guidelines [8] on physical restraint for critically ill adults. We will engage key stakeholders and knowledge users including ICU survivors and family members in the development of these documents and to inform our knowledge translation strategies. We will also engage these individuals in the identification of research gaps and priorities for research in relation to physical restraint of critically ill patients. We anticipate our systematic review and subsequent guideline development will foster patient and clinician safety by providing clinicians, administrators, and policymakers with a practical and evidence-based tool to promote minimal and safe use of physical restraint for critically ill adults.

\section{Additional files}

Additional file 1: PRISMA-P checklist. PRISMA-P checklist. (DOCX $35 \mathrm{~kb}$ ) Additional file 2: Search strategies_MEDLINE. Medline search strategy. (DOCX $14 \mathrm{~kb}$ )

Additional file 3: CERQual Qualitative Evidence Profile. CERQual Qualitative Evidence Profile. (DOCX 14 kb)

\section{Abbreviations}

CASP: Critical Appraisal Skills Programme; CERQual: Confidence in the Evidence from Reviews of Qualitative Research; Cl: confidence interval; GRADE: Grading of Recommendations Assessment, Development and Evaluation; ICU: Intensive care unit; PRESS: Peer Review for Electronic Search Strategies; PRISMA: Preferred Reporting Items for Systematic Reviews and Meta-Analyses; RR: risk ratio; SD: standrad deviation; SIGN: Scottish Intercollegiate Guidelines Network; US: United States

\section{Acknowledgements}

We would like to acknowledge Becky Skidmore, Information Specialist (Systematic Reviews), for her assistance in the design and execution of the search strategy.

\section{Funding}

This review has not received funding and there is no sponsor. Dr. Louise Rose holds a CIHR New Investigator Award.

\section{Authors' contributions}

$L R$ is responsible for conceiving the review, designing the first draft of the review protocol, refinement of the clinical question and methodologies, coordinating the review, reference screening, data extraction and management, statistical inferences, interpretation of the data, and writing the first draft of the manuscript and is the guarantor of the review. CD is responsible for the refinement of the clinical question and methodologies, data extraction, interpretation of the data, and editorial comments on the manuscript drafts. OS is responsible for the refinement of the clinical question and methodologies, data extraction, interpretation of the data, and editorial comments on the manuscript drafts. LB is responsible for the refinement of the clinical question and methodologies, data extraction, interpretation of data, and editorial comments on the manuscript drafts. GE is responsible for the refinement of the clinical question and methodologies, reference screening, interpretation of the data, and editorial comments on the manuscript drafts. DF is responsible for the refinement of the clinical question and methodologies, statistical inferences, interpretation of the data, and editorial comments on the manuscript drafts. SS is responsible for the refinement of the clinical question and methodologies, data extraction, interpretation of the data, and editorial comments on the manuscript drafts. LW is responsible for the refinement of the clinical question and methodologies, data extraction, interpretation of the data, and editorial comments on the manuscript drafts. TS is responsible for the refinement of the clinical question and methodologies, data extraction, interpretation of the data, and editorial comments on the manuscript drafts. SM is responsible for conceiving the review, designing the first draft of the review protocol, refinement of the clinical question and methodologies, interpretation of the data, and writing the first draft of the manuscript. All authors read and approved the manuscript. 


\section{Consent for publication}

Not applicable.

\section{Ethics approval and consent to participate} Not applicable.

\section{Registration}

This systematic review is registered with PROSPERO, an international prospective register of systematic reviews: http://www.crd.york.ac.uk/ PROSPERO/display_record.asp?ID=CRD42015027860

\section{Author details}

'Department of Critical Care Medicine, Sunnybrook Health Sciences Centre, 2075 Bayview Ave, Toronto M4N 3M5, Canada. 'Lawrence S. Bloomberg Faculty of Nursing, University of Toronto, 155 College St., Toronto M5T 1P8, Canada. ${ }^{3}$ Institute for Clinical Evaluative Sciences, Veterans Hill Trail, 2075 Bayview Avenue G1 06, Toronto M4N 3M5, Canada. ${ }^{4}$ Provincial Centre of Weaning Excellence, Michael Garron Hospital, 825 Coxwell Ave, East York M4C 3E7, Canada. ${ }^{5}$ Department of Critical Care, Nursing/Clinical Research, Li Ka Shing Knowledge Institute, St. Michael's Hospital, 30 Bond St, Toronto, ON M5B 1W8, Canada. 'Leslie Dan Faculty of Pharmacy, University of Toronto, 144 College St, Toronto M5S 3M2, Canada. ${ }^{7}$ Mount Sinai Hospital, 600 University Ave, Toronto M5G 1X5, Canada. ${ }^{8}$ Clinical Epidemiology Program, Ottawa Hospital Research Institute, 500 Smyth Road, Ottawa, ON K1H 8L6, Canada. ${ }^{9}$ Faculty of Medicine, University of Ottawa, 451 Smyth Rd, Ottawa K1H 8M5, Canada. ${ }^{10}$ Faculty of Medicine, University of Toronto Medical Sciences Building, 1 King's College Cir \#3172, Toronto M5S 1A8, Canada. ${ }^{11}$ Department of Family and Community Medicine, 500 University Ave, Toronto M5G 1V7, Canada. ${ }^{12}$ Institute of Health Policy, Management and Evaluation, University of Toronto, 155 College St, Toronto, ON M5T 3M6, Canada.

\section{Received: 18 August 2016 Accepted: 1 November 2016}

\section{Published online: 21 November 2016}

\section{References}

1. Mehta S, Burry L, Cook D, Fergusson D, Steinberg M, Granton J, et al. A randomized trial of daily sedation interruption in mechanically ventilated critically ill patients cared for with a sedation protocol. JAMA. 2012;308:1985-92.

2. Adhikari N, Fowler R, Bhagwanjee S, Rubenfeld G. Critical care and the global burden of critical illness in adults. Lancet. 2010;376:1339-46.

3. Shahin J, Harrison D, Rowan K. Is the volume of mechanically ventilated admissions to UK critical care units associated with improved outcomes? Intensive Care Med. 2014;40:353-60.

4. Wunsch H, Wagner J, Herlim M, Chong D, Kramer A, Halpern S. ICU occupancy and mechanical ventilator use in the United States. Crit Care Med. 2013:41:2712-9.

5. Benbenbishty J, Adam S, Endacott R. Physical restraint use in intensive care units across Europe: the PRICE study. Intensive Crit Care Nurs. 2010;26:241-5.

6. Rose L, Burry L, Mallick R, Luk E, Cook D, Fergusson D, et al. Prevalence, risk factors, and outcomes associated with physical restraint use in mechanically ventilated adults. J Crit Care. 2016;31:31-5.

7. Registered Nurses' Association of Ontario. Promoting safety: alternative approaches to the use of restraints. Toronto: Registered Nurses' Association of Ontario; 2012

8. Maccioli G, Dorman T, Brown B, Mazuski J, McLean B, Kuszaj J, et al. Clinical practice guidelines for the maintenance of patient physical safety in the intensive care unit: use of restraining therapies-American College of Critical Care Medicine Task Force 2001-2002. Crit Care Med. 2003:11:2665-76.

9. Chang L, Wang K, Chao Y. Influence of physical restraint on unplanned extubation of adult intensive care patients: a case-control study. Am J Crit Care. 2008;17:408-15.

10. Curry K, Cobb S, Kutash M, Diggs C. Characteristics associated with unplanned extubations in a surgical intensive care unit. Am J Crit Care. 2008;17:45-51. quiz 2.

11. Tung A, Tadimeti L, Caruana-Montaldo B, Atkins PM, Mion LC, Palmer RM, et al. The relationship of sedation to deliberate self-extubation. J Clin Anesth. 2001:13:24-9.

12. Mion L, Minnick A, Leipzig R, Catrambone C, Johnson M. Patient-initiated device removal in intensive care units: a national prevalence study. Crit Care Med. 2007;35:2714-20.
13. Happ MB. Treatment interference in acutely and critically ill adults. Am J Crit Care. 1998:7:224-35.

14. Birkett KM, Southerland KA, Leslie GD. Reporting unplanned extubation. Intensive Crit Care Nurs. 2005:21:65-75.

15. Atkins PM, Mion LC, Mendelson W, Palmer RM, Slomka J, Franko T. Characteristics and outcomes of patients who self-extubate from ventilatory support: a case-control study. Chest. 1997;112:1317-23.

16. Chuang M, Lee C, Chen Y, Huang S, Lin I. Revisiting unplanned endotracheal extubation and disease severity in intensive care units. PLoS One. 2015;10:e0139864.

17. Evans D, Wood J, Lambert L. Patient injury and physical restraint devices: a systematic review. J Adv Nurs. 2003;41:274-82.

18. Van Rompaey B, Elseviers M, Schuurmans M, Shortridge-Baggett L, Truijen S, Bossaert L. Risk factors for delirium in intensive care patients: a prospective cohort study. Crit Care. 2009;13:R77

19. Micek S, Anand N, Laible B, Shannon W, Kollef M. Delirium as detected by the CAM-ICU predicts restraint use among mechanically ventilated medical patients. Crit Care Med. 2005;33:1260-5

20. Mehta S, Cook D, Devlin J, Skrobik Y, Meade M, Fergusson D, et al. Incidence, risk factors and outcomes of delirium in mechanically ventilated adults. Crit Care Med. 2015:43:557-66.

21. Hatchett C, Langley G, Schmollgruber S. Psychological sequelae following ICU admission at a level 1 academic South African hospital. South Afr J Crit Care. 2010;26:52-8.

22. Jones C, Backman C, Capuzzo M, Flaatten H, Rylander C, Griffiths R. Precipitants of post-traumatic stress disorder following intensive care: a hypothesis generating study of diversity in care. Intensive Care Med. 2007;33:978-85.

23. Kandeel N, Attia A. Physical restraints practice in adult intensive care units in Egypt. Nurs \& Health Sci. 2013;15:79-85.

24. Baumgarten M, Margolis D, Localio A, Kagan S, Lowe R, Kinosian B, et al. Extrinsic risk factors for pressure ulcers early in the hospital stay: a nested case-control study. J Gerontol A Biol Sci Med Sci. 2008;63:408-13.

25. Government of Ontario: Patient restraints minimization act. Canada: http:// www.e-laws.gov.on.ca/html/statutes/english/elaws_statutes_01p16_e.htm; 2001 Accessed 20 Jun 2015.

26. Bray K, Hill K, Robson W, Leaver G, Walker N, O'Leary M, et al. British Association of Critical Care Nurses position statement on the use of restraint in adult critical care units. Nurs Crit Care. 2004;9:199-212.

27. Shamseer L, Moher D, Clarke M, Ghersi D, Liberati A, Petticrew M, et al. Preferred reporting items for systematic review and meta-analysis protocols (PRISMA-P) 2015: elaboration and explanation. BMJ. 2015;349:g7647.

28. McGowan J, Sampson M, Salzwedel DM, Cogo E, Foerster V, Lefebvre C. PRESS Peer Review of Electronic Search Strategies: 2015 Guideline Statement. J Clin Epidemiol. 2016;75:40-6.

29. Sampson M, McGowan J, Cogo E, Grimshaw J, Moher D, Lefebvre C. An evidence-based practice guideline for the peer review of electronic search strategies. J Clin Epidemiol. 2009;62:944-52.

30. Arai L, Roen K, Roberts H, Popay J. It might work in Oklahoma but will it work in Oakhampton? Context and implementation in the effectiveness literature on domestic smoke detectors. Inj Prev. 2005;11:148-51.

31. Mays N, Pope C, Popay J. Systematically reviewing qualitative and quantitative evidence to inform management and policy-making in the health field. J Health Serv Res Pol. 2005;10:6-20.

32. Popay J. Moving beyond floccinaucinihilipilification: enhancing the utility of systematic reviews. J Clin Epidemiol. 2005;58:1079-80.

33. EndNote. X7 edition. Carlsbad, CA, USA: Thomson Reuters; 2015.

34. Moher D, Liberati A, Tetzlaff J, Altman DG, Group P. Preferred reporting items for systematic reviews and meta-analyses: the PRISMA statement. PLoS Med. 2009;6:e1000097.

35. Glenton C, Colvin C, Carlsen B, Swartz A, Lewin S, Noyes J, et al. Barriers and facilitators to the implementation of lay health worker programmes to improve access to maternal and child health: qualitative evidence synthesis. Cochrane Database Syst Rev. 2013;8:CD010414.

36. Higgins JPT, Green S, editors. Cochrane Handbook for Systematic Reviews of Interventions 5.1.0 [updated March 2011]. The Cochrane Collaboration; 2011. Available from http://handbook.cochrane.org.

37. Bai A, Shukla V, Bak G, Wells G. Quality assessment tools project report. Ottawa: Canadian Agency for Drugs and Technologies for Health; 2012.

38. Hannes K. Chapter 4: Critical appraisal of qualitative research. In: Noyes J, Booth A, Hannes K, Harden A, Harris J, Lewin S, Lockwood C, editors. Supplementary Guidance for Inclusion of Qualitative Research in Cochrane 
Systematic Reviews of Interventions. Cochrane Collaboration Qualitative Methods Group; 2011

39. 10 questions to help you make sense of qualitative research http://media.wix.com/ ugd/dded87_29c5b002d99342f788c6ac670e49f274.pdf. Accessed 31 May 2016

40. Popay J, Rogers A, Williams G. Rationale and standards for the systematic review of qualitative literature in health services research. Qual Health Res. 1998;:8:341-51

41. The PRISMA flow diagram [http://www.prisma-statement.org/ PRISMAStatement/FlowDiagram.aspx] Accessed 31 May 2016

42. Vaismoradi $\mathrm{M}$, Turunen $\mathrm{H}$, Bondas T. Content analysis and thematic analysis: implications for conducting a qualitative descriptive study. Nurs Health Sci. 2013;15:398-405.

43. Hozo SP, Djulbegovic B, Hozo I. Estimating the mean and variance from the median, range, and the size of a sample. BMC Med Res Methodol. 2005;5:13.

44. Higgins JP, Thompson SG. Quantifying heterogeneity in a meta-analysis. Stat Med. 2002;21:1539-58.

45. Egger M, Davey Smith G, Schneider M, Minder C. Bias in meta-analysis detected by a simple, graphical test. BMJ. 1997;315:629-34.

46. Macaskill P, Walter SD, Irwig L. A comparison of methods to detect publication bias in meta-analysis. Stat Med. 2001;20:641-54.

47. Peters JL, Sutton AJ, Jones DR, Abrams KR, Rushton L. Comparison of two methods to detect publication bias in meta-analysis. JAMA. 2006;295:676-80

48. Guyatt GH, Oxman AD, Vist GE, Kunz R, Falck-Ytter Y, Alonso-Coello P, et al. GRADE: an emerging consensus on rating quality of evidence and strength of recommendations. BMJ. 2008;336:924-6.

49. Lewin S, Glenton C, Munthe-Kaas H, Carlsen B, Colvin C, Gülmezoglu M, et al. Using qualitative evidence in decision making for health and social interventions: an approach to assess confidence in findings from qualitative evidence syntheses (GRADE-CERQual). PLoS Med. 2015;12:e1001895.

50. Minnick AF, Mion LC, Johnson ME, Catrambone C, Leipzig R. Prevalence and variation of physical restraint use in acute care settings in the US. J Nurs Scholarsh. 2007;39:30-7.

51. De Jonghe B, Constantin J, Chanques G, Capdevila X, Lefratn J, Outin H, et al. Physical restraint in mechanically ventilated ICU patients: a survey of French practice. Intensive Care Med. 2013;39:31-7.

52. Martin B, Mathisen L. Use of physical restraints in adult critical care: a bicultural study. Am J Crit Care. 2005;14:133-42.

\section{Submit your next manuscript to BioMed Central and we will help you at every step:}

- We accept pre-submission inquiries

- Our selector tool helps you to find the most relevant journal

- We provide round the clock customer support

- Convenient online submission

- Thorough peer review

- Inclusion in PubMed and all major indexing services

- Maximum visibility for your research

Submit your manuscript at www.biomedcentral.com/submit
Biomed Central 